\title{
The Ratio of the Perimeter and the Area of Unions of Copies of a Fixed Set
}

\section{Z. Gyenes}

Received: 30 March 2008 / Revised: 13 November 2010 / Accepted: 15 November 2010 /

Published online: 7 January 2011

(C) Springer Science+Business Media, LLC 2011

\begin{abstract}
T. Keleti asked 10 years ago whether the ratio of the perimeter and the area of a union of unit squares is always at most 4 . In the present paper we show that the ratio is less than 5.6.
\end{abstract}

Keywords Inverse isoperimetric inequality $\cdot$ Integral geometry $\cdot$ Extremal problems in discrete geometry

\section{Introduction}

Tamás Keleti proved that if we take a finite union of unit squares, the ratio of the perimeter and area of the resulting figure cannot be arbitrarily large [2, Remark 14, p. 115]. (This statement was also posed as Problem 6 of the 1998 Schweitzer Contest in Higher Mathematics [3].) Keleti asked whether or not 4 is a good upper bound. The bounds obtained by Keleti in [2] and the contestants of the Schweitzer competition were very far from the conjectured ratio 4 . In this paper we give a new proof for the theorem which gives an improved bound that is slightly less than 5.6.

For every bounded measurable plane set $E$, let $p(E)$ and $a(E)$ denote the perimeter (linear measure of the boundary $\partial E$ ) and the area (Lebesgue measure) of $E$. We put $r(E)=p(E) / a(E)$ if $a(E)>0$. Keleti's question can be generalized as follows: is it true that for any compact $U$ with $a(U)>0$, if $S$ is a finite union of congruent copies of $U$, then $r(S) \leq r(U)$ ? The following example shows that this is false even for some triangles.

Let $\Delta a b c$ be an isosceles triangle with sides $a b$ and $a c$ of length 1 and base $b c$ of length $\varepsilon$. For $\varepsilon$ small, $r(\Delta a b c)$ is about $4 / \varepsilon$. Now take the circumscribed circle of

Research was supported by the Hungarian National Foundation, Grant No K72655.

Z. Gyenes $(\bowtie)$

Department of Analysis, Eötvös Loránd University, Pázmány Péter sétány 1/C 1117, Budapest,

Hungary

e-mail: zol@cs.elte.hu 
$\triangle a b c$ and dilate it from the point $a$ with ratio $1+\varepsilon$, and let $O$ denote the center of this dilated circle. Rotate $\Delta a b c$ about $O$ by the angles $2 k \pi / N(k=1, \ldots, N)$. Let $S$ be the union of these rotated triangles. The area of $S$ is about the area of a circle of radius $1 / 2$, while for a large enough $N$, its perimeter is about $2 / \varepsilon$ times the perimeter of a circle with radius $1 / 2$, so $r(S)$ is about $8 / \varepsilon$, and we have seen that this is about $2 r(\Delta a b c)$.

For every compact set $U$ with $a(U)>0$, we shall denote by $C_{U}$ the smallest constant $C$ for which $r(S) \leq C \cdot r(U)$ holds whenever $S$ is a finite union of congruent copies of $U$. (We write $C_{U}=\infty$ if there is no such $C$.) We shall see that $C_{U}$ is finite whenever $U$ is a "nice" Jordan domain (Theorem 4.2). We shall say that a Jordan domain is nice if its boundary is piecewise $C^{1}$ (with respect to the arc-length parameterization) and has no cusp points.

As the example above shows, $C_{U}>1$ can happen even for some convex $U$. Keleti's question is equivalent to $C_{Q}=1$ when $Q$ is a unit square. This question is still unsolved. In fact, we do not know any compact set $U$ satisfying $C_{U}=1$, except the disc (see Corollary 2.7).

We may ask whether or not $C_{U}$ is bounded by an absolute constant for every nice Jordan domain $U$. The answer is negative, as the following example shows. For a given positive integer $n>1$, let $U_{n}$ be the union of a unit disc with center $O$ and an isosceles triangle $\triangle a b c$ such that $a$ and $b$ are boundary points of the unit disc, $c$ is chosen outside the unit disc, $|c-a|=|c-b|=1 / n$, and $|a-b|=1 / n^{2}$. Now $r\left(U_{n}\right) \rightarrow 2$ as $n \rightarrow \infty$. Rotate $U_{n}$ about $O$ by $2 k \pi / N(k=1, \ldots, N)$ and take the union of these sets. For large enough $N$, the perimeter of the union becomes approximately $2 \pi n$, while the area of it tends to $\pi$. Thus, $C_{U_{n}} \rightarrow \infty$ as $n \rightarrow \infty$.

The question whether or not $C_{U}$ is bounded for convex compact sets $U$ remains open. In Theorem 5.3 we shall present an upper bound of $C_{U}$ depending on the shape of $U$.

The basic ingredient of our method is an integral-geometric formula for the area of polygons using line integrals of certain averages of distances.

\section{Some General Formulas and Estimates}

Definition 2.1 Let $S$ be a compact plane set which is the closure of its interior, and let $q \in S$. For every $\phi \in[0,2 \pi)$, let $z_{\phi}$ be the unique point of $S$ such that the line segment with endpoints $q$ and $z_{\phi}$ is contained in $S$, the angle between the vector $\overrightarrow{q z_{\phi}}$ and the positive $x$ axis equals $\phi$, and $\overrightarrow{q z_{\phi}}$ has the longest possible length. Let $v^{S}(q, \phi)$ denote the vector $\overrightarrow{q z_{\phi}}$. The average of the vector-valued function $v^{S}(q, \phi)$ is defined as

$$
v^{S}(q):=\frac{1}{2 \pi} \int_{0}^{2 \pi} v^{S}(q, \phi) d \phi .
$$

The following two lemmas establish the connection between the previously defined functions and the area of $S$.

The boundary of a set $S$ is denoted by $\partial S$. We shall denote by $\partial^{*} S$ the set of smooth points of $\partial S$. A point of the boundary is smooth if its intersection with a small disk around the point is a $C^{1}$ arc (using the arc-length parameterization). 
Let $q \in \partial^{*} S$. We denote by $n^{S}(q)$ the outer normal vector at $q$; that is, $n^{S}(q)$ is the unit vector directed outward and is orthogonal to the boundary of $S$ at $q$. Furthermore, let $|\cdot|$ denote the usual arc-length metric on $\partial S$.

By a polygon we mean a compact plane set which is the closure of its interior and whose boundary consists of finitely many line segments.

With this notation, the area of a polygon can be expressed with a contour integral:

Lemma 2.2 Let $S$ be a polygon, and let $\phi \in[0,2 \pi)$ be fixed. Then the area of $S$ is $-\oint_{\partial S}\left\langle v^{S}(q, \phi), n^{S}(q)\right\rangle|d q|$.

Proof By choosing an appropriate coordinate system we may assume that $\phi=0$. We would like to apply the Gauss-Ostrogradsky theorem to rewrite the integral as

$$
\iint_{S} \frac{\partial v_{1}^{S}(q, 0)}{\partial x}+\frac{\partial v_{2}^{S}(q, 0)}{\partial y} d x d y,
$$

where $q=(x, y)$, and $v_{1}^{S}(q, 0)$ and $v_{2}^{S}(q, 0)$ are the coordinates of $v^{S}(q, 0)$. Unfortunately, the vector field $v^{S}(q, 0)$ is usually not $C^{1}$, so we shall divide $S$ into smaller pieces such that $v^{S}(q, 0)$ is a $C^{1}$ vector field in each of the pieces. This can be done by slicing up $S$ with horizontal lines going through the vertices of $S$. Note that the contour integral on these smaller sets give 0 on the parts of their boundaries not contained in the boundary of $S$ (since these are horizontal line segments), so the original contour integral is the sum of the contour integrals on the smaller pieces.

Since $\frac{\partial v_{1}^{S}(q, 0)}{\partial x}=-1$ and $\frac{\partial v_{2}^{S}(q, 0)}{\partial y}=0$, the contour integral on each piece equals -1 times the area of the piece, and thus the integral is

$$
\iint_{S}-1 d x d y=-a(S)
$$

This proves the lemma.

Lemma 2.3 For every polygon $S$, we have

$$
a(S)=-\oint_{\partial S}\left\langle v^{S}(q), n^{S}(q)\right\rangle|d q| .
$$

Proof First, we claim that if $q$ is on the boundary of $S$, then $\left\langle v^{S}(q, \phi), n^{S}(q)\right\rangle$ is nonpositive. Indeed, either $v^{S}(q, \phi)$ is the zero vector, and then our claim is obvious, or it is a nonzero vector, but then the angle of the vectors $v^{S}(q, \phi)$ and $n^{S}(q)$ is not acute, and so their scalar product is again nonpositive.

By the definition of $v^{S}(q)$, the right-hand side of (1) equals

$$
-\frac{1}{2 \pi} \oint_{\partial S} \int_{0}^{2 \pi}\left\langle v^{S}(q, \phi) d \phi, n^{S}(q)\right\rangle|d q| .
$$


By Fubini's theorem, the order of integration can be changed, since $\left\langle v^{S}(q, \phi), n^{S}(q)\right\rangle$ is nonpositive. So one can rewrite the expression as

$$
-\frac{1}{2 \pi} \int_{0}^{2 \pi}\left(\oint_{\partial S}\left\langle v^{S}(q, \phi), n^{S}(q)\right\rangle|d q|\right) d \phi .
$$

Now it is enough to show that the inner integral equals $-a(S)$ for all fixed direction $\phi$, and this is what we proved in Lemma 2.2.

We remark that in the definition of $v^{S}(q)$ we could have integrated with respect to an arbitrary Borel measure instead of the Lebesgue measure. It is easy to see that Lemma 2.3 remains valid for these more general averages $v^{S}(q)$. On the other hand, in the next lemma it is important that we are using the Lebesgue measure in the definition of $v^{S}(q)$.

Lemma 2.4 Suppose that $S$ is a polygon and $-\left\langle v^{S}(q), n^{S}(q)\right\rangle \geq m>0$ for every $q \in \partial^{*} S$. Then $r(S) \leq 1 / m$.

Proof By Lemma 2.3, $a(S)=-\oint_{\partial S}\left\langle v^{S}(q), n^{S}(q)\right\rangle|d q|$, so using the assumption, we get $a(s) \geq \oint_{\partial S} m|d q|$, which gives $a(s) \geq m \cdot p(S)$, so dividing by $p(S)$ we get the inequality.

Lemma 2.5 Suppose that $S$ and $U$ are polygons, $U \subset S$, and $q \in \partial^{*} S \cap \partial^{*} U$. Then

$$
-\left\langle v^{S}(q), n^{S}(q)\right\rangle \geq-\left\langle v^{U}(q), n^{U}(q)\right\rangle
$$

Proof By $q \in \partial^{*} S \cap \partial^{*} U$, we have $n^{S}(q)=n^{U}(q)$. Now $U$ is contained in $S$, so $\left|v^{S}(q, \phi)\right| \geq\left|v^{U}(q, \phi)\right|$ for every $0 \leq \phi<2 \pi$. The angle between the vectors $n^{S}(q)$ and $v^{S}(q, \phi)$ and similarly $\bar{n}^{U}(q)$ and $v^{U}(q, \phi)$ is at least $\pi / 2$, so $-\left\langle v^{S}(q, \phi), n^{S}(q)\right\rangle \geq-\left\langle v^{U}(q, \phi), n^{U}(q)\right\rangle$ also holds, and then integrating by $\phi$, we get $-\left\langle v^{S}(q), n^{S}(g)\right\rangle \geq\left\langle v^{U}(q), n^{U}(q)\right\rangle$.

We recall that $r(U)$ denotes the ratio of the perimeter and the area of $U$, while $C_{U}$ was the supremum of the ratios $r(S) / r(U)$, where $S$ is a finite union of copies of $S$.

Lemma 2.6 For every polygon $U$, we have

$$
C_{U} \leq \frac{1}{r(U) \cdot \inf \left\{-\left\langle v^{U}(q), n^{U}(q)\right\rangle: q \in \partial^{*} U\right\}} .
$$

Proof This is clear from Lemmas 2.4 and 2.5.

Corollary 2.7 For every disc $U$, we have $C_{U}=1$.

Proof From the rotational symmetry of the disc it follows that $\left\langle v^{U}(q), n^{U}(q)\right\rangle$ is constant. Applying Lemma 2.3, we get that $\left\langle v^{U}(q), n^{U}(q)\right\rangle=-a(U) / p(U)=-1 / r(U)$ for every $q \in \partial U$. By Lemma 2.6 we are done. 


\section{Unions of Squares}

Theorem 3.1 If $S$ is a finite union of unit squares (having arbitrary position in the plane), then the ratio of the perimeter and area of $S$ is at most $\frac{2 \pi}{\frac{1}{2} \log 2+\frac{\pi}{4}}<5.551$.

Lemma 3.2 Let $U$ be a unit square and suppose that $q \in \partial^{*} U$ is a smooth point. Then

$$
-\left\langle v^{U}(q), n^{U}(q)\right\rangle \geq \frac{\frac{1}{2} \log 2+\frac{\pi}{4}}{2 \pi} .
$$

Proof Suppose that $q$ divides the appropriate side of $U$ into line segments of lengths $1-x$ and $x(0<x<1)$. Then an easy calculation gives

$$
\begin{aligned}
-2 \pi \cdot\left\langle d^{U}(q), n^{U}(q)\right\rangle= & \int_{0}^{\arctan \frac{1}{x}} x \tan \phi d \phi+\int_{\arctan \frac{1}{x}}^{\pi-\arctan \frac{1}{1-x}} 1 d \phi \\
& +\int_{\pi-\arctan \frac{1}{1-x}}^{\pi}(1-x) \tan (\pi-\phi) d \phi \\
= & \frac{1}{2} x \log \left(1+\frac{1}{x^{2}}\right)+\left(\pi-\arctan \frac{1}{x}-\arctan \frac{1}{1-x}\right) \\
& +\frac{1}{2}(1-x) \log \left(1+\frac{1}{(1-x)^{2}}\right) .
\end{aligned}
$$

Another easy calculation shows that for $0<x<1$, the right-hand side is convex from above and has limits $\frac{1}{2} \log 2+\frac{\pi}{4}$ at the points $x=0$ and $x=1$. This finishes the proof of the inequality.

Now Theorem 3.1 is an easy consequence of the lemmas of the previous section. Indeed, if $U$ is a unit square, then Lemma 3.2 gives the lower bound $m=\frac{\frac{1}{2} \log 2+\frac{\pi}{4}}{2 \pi}$. Then, by Lemma 2.5 , this is also a lower bound for the union $S$. Finally, by Lemma 2.4, we obtain $r(S) \leq \frac{2 \pi}{\frac{1}{2} \log 2+\frac{\pi}{4}}$.

\section{Unions of Other Sets}

In this section we investigate the case where the unit square is replaced by other sets. So the question is the following: if we take a union of sets that are congruent to a given set of the plane, is it true that the ratio of its perimeter and area is bounded from above by a constant depending on the given set? First, we note the following.

Proposition 4.1 Let E be a plane set which is a finite union of polygons congruent to a given polygon $U$. Then $r(E)$ is bounded from above by a constant only depending on $U$. That is, $C_{U}$ is finite for every polygon $U$. 
Proof We shall use Lemmas 2.4 and 2.5. Since every polygon $U$ can be obtained as a finite union of congruent triangles (the triangles in the union can have common inner points), it is enough to find a bound $m$ in the inequality of Lemma 2.4 for triangles. Let $T=\triangle a b c$ denote our triangle. At each vertex of the triangle, we consider both sides incident to the chosen vertex as a supporting line and calculate $-\left\langle v^{T}(q), n^{T}(q)\right\rangle$ for the vertices of $T$, where $n^{T}(q)$ is chosen according to the considered supporting line. This way we get six possible positive values for $-\left\langle v^{T}(q), n^{T}(q)\right\rangle$ for the vertices of the triangle with the chosen supporting lines. We prove that the smallest of these six values is a good lower bound for all $-\left\langle v^{T}(q), n^{T}(q)\right\rangle$, where $q$ is an arbitrary point of $\partial T$. Now let $q$ be a point of the side $a b$, and let $x$ denote the ratio $|a-q| /|a-b|$. Let us draw lines through $q$ which are parallel to $a c$ and $b c$, and cut out the parallelogram that is bounded by $a c, b c$, and these two lines. Let $T^{\prime}$ denote the remaining figure. An easy calculation shows that

$$
\begin{aligned}
-\left\langle v^{T}(q), n^{T}(q)\right\rangle & \geq-\left\langle v^{T^{\prime}}(q), n^{T}(q)\right\rangle \\
& =-\left(x \cdot\left\langle v^{T}(b), n^{T}(q)\right\rangle+(1-x) \cdot\left\langle v^{T}(a), n^{T}(q)\right\rangle\right),
\end{aligned}
$$

which proves that the smallest of the six values we get at the vertices is a good choice for $m$ in the inequality of Lemma 2.4, so we are done.

In the general case we have to assume that the given set is "nice" enough: it is natural to require that the set is closed and that its boundary is a rectifiable Jordan curve. It is easy to prove that this requirement is still not sufficient: if the set has cusp points on its boundary (by cusp point we mean a point where the left and the right derivatives by the arc-length parameterization are opposite vectors), then such a bound does not exist. In this section we shall consider Jordan domains with piecewise $C^{1}$ boundary (with respect to the arc-length parameterization) and without cusp points.

We shall call these sets nice Jordan domains. In the next theorem we prove that for arbitrary unions of congruent copies of a given nice Jordan domain, the ratio $r=$ perimeter/area is bounded from above.

Theorem 4.2 Let $U$ be a nice Jordan domain. If the bounded set $E$ is the union of copies of $U$, then $r(E) \leq C \cdot r(U)$, where $C$ is a constant only depending on $U$. In particular, $C_{U}<\infty$ for every nice Jordan domain $U$.

Lemma 4.3 Let $U$ be a nice Jordan domain. Then $U$ is a union of congruent triangles.

Proof For every boundary point $q \in \partial U$, let $\phi(q)$ denote the angle of the two halflines tangent to $\partial U$ and starting from $q$. Since $U$ is nice, we have $\phi(q)=\pi$ except for finitely many points and, since there are no cusp points, $\phi(q)>0$ for every $q \in \partial U$. Let $\phi_{0}=\min \{\phi(q): q \in \partial U\}$.

Let $T_{n}$ denote the isosceles triangle with legs of length $1 / n$ and having angle $1 / n$ opposite to the base. We prove that there exists a positive integer $n$ such that every point of $U$ is covered by a congruent copy of $T_{n}$ contained in $U$. 
Suppose that such an $n$ does not exist. Then, for each positive integer $n$, there exists a point $x_{n} \in U$ not covered by any congruent copy of $T_{n}$ contained in $U$. Since $U$ is compact, we can choose a convergent subsequence $x_{n_{k}}$ of $x_{n}$.

It is clear that the limit $q$ of the sequence $x_{n_{k}}$ cannot be an inner point of $U$. This means that $q \in \partial U$. We may choose an appropriate coordinate system where $q$ is the origin, and a small subarc $\gamma$ of $\partial U$ around $q$ equals the graph of a Lipschitz function $f:[-\delta, \delta] \rightarrow \mathbb{R}$. Let $\gamma^{\prime}$ denote the graph of $f$ above $[-\delta / 2, \delta / 2]$. Since $\gamma^{\prime}$ has positive distance from $\partial U \backslash \gamma$, it is clear that there is a neighborhood $V$ of $\gamma^{\prime}$ and there is an $n_{0}$ such that each point of $U \cap V$ is covered by a congruent copy of $T_{n}$ contained in $U$ for every $n>n_{0}$. As $x_{n_{k}} \in U \cap V$ for every $k$ large enough, this is a contradiction.

Lemma 4.4 Let $T$ be a polygon. If $E$ is a bounded set and $E$ is the union of (possibly infinitely many) congruent copies of $T$, then $E$ is measurable, and $r(E) \leq C_{T} \cdot r(T)$.

Remark 4.5 Note that $C_{T}$ was defined for only finite unions. This lemma shows that all our theorems remain valid with the same constants if we take away the condition that the unions must be finite.

Proof The proof of the lemma is based on some ideas from [1].

Suppose that an $\varepsilon<\operatorname{diam} T$ is given. Our aim is to find a suitable covering of $\partial E$ with sets having diameter at most $\varepsilon$ (we shall call such coverings $\varepsilon$-coverings). By the definition of the one-dimensional Hausdorff measure, what we need to prove is that the sum of the diameters of the covering sets is at most $C_{T} \cdot r(T)$. For an arbitrary but fixed positive $v$ and each point $x \in \partial E$, there exists a set $T_{x} \subset E$ congruent to $T$ with dist $\left(x, T_{x}\right)<v / 2$. Since $\partial E$ is compact, it can be covered by finitely many balls $B\left(x_{i}, v / 2\right)$, where $x_{1}, \ldots, x_{k} \in \partial E$. This implies that the $v$-neighborhood of $\partial\left(\bigcup_{i=1}^{k} T_{x_{i}}\right)$ contains $\partial E$.

The boundary of the set $E_{v}=\bigcup_{i=1}^{k} T_{x_{i}}$ is a disjoint union of connected components. These components can be obtained as finite unions of line segments in such a way that the intersection of any two of these line segments is either empty or is a common endpoint of the segments. We consider these components as graphs: the edges of the graphs are the line segments, and the vertices are the endpoints. Each vertex of these graphs has an even degree, since the regions between adjacent edges starting from the same vertex alternately belong to $E_{v}$ and the complement of $E_{v}$. Consequently, all such graphs contain an Euler walk (i.e., a walk on the edges which visits each edge exactly once).

From the above it can be deducted that each of the components of $\partial E_{v}$ of length $l \geq \varepsilon$ admits an $\varepsilon$-covering with at most $2 l / \varepsilon$ sets with total diameter at most $l$ (we divide the Euler walk into $[l / \varepsilon]+1$ parts of equal length). We will need this fact in the last paragraph of the proof.

Now take a connected component $G$ of $\partial E_{v}$ shorter than $\varepsilon$. Let $C$ be a cycle in the graph corresponding to $G$. First, we show that the interior of $C$ as a Jordan curve cannot contain any point of $E_{v}$. Indeed, if it contained a point from $E_{v}$, then it would also contain one of the polygons in $E$ congruent to $T$, and this is impossible since, by the assumption $\varepsilon<\operatorname{diam} T$, the cycle is shorter than the diameter of the polygon. 
Since every vertex of our graph has an even degree, $G$ is an edge-disjoint union of cycles, and the interior of these cycles cannot contain any point from $E_{v}$. Thus, a small neighborhood of $G$ minus the interiors of the cycles must be contained in $E_{v}$.

Now we prove that for every point $x$ in the complement of $E_{v}$, one of the following statements is true. Either the point $y \in E_{v}$ nearest to $x$ belongs to a connected component of $\partial E_{v}$ of length at least $\varepsilon$, or $x$ is contained in the interior of a cycle in a connected component shorter than $\varepsilon$. Indeed, we have to show that if $y$ belongs to a connected component shorter than $\varepsilon$, then $x$ is in the interior of the cycles of the graph corresponding to the component. Apart from the endpoints, the line segment $[x, y]$ must be disjoint from $E_{v}$, so by what we proved above, $x$ must be contained in the interior of one of the cycles.

After these preparations we can construct a "good" $(\varepsilon+v)$-covering of $\partial E$. First, we take $\varepsilon$-coverings of the connected components of $\partial E_{v}$ of length at least $\varepsilon$ such that the total number of the sets covering these connected components is at most $2 \cdot p\left(E_{v}\right) / \varepsilon$, and the sum of the diameters of these sets is at most the total length of these connected components. Take the $v$-neighborhood of the sets of this covering. From the previously proved facts it is clear that these "inflated" sets together with the interior of the cycles in the connected components shorter than $\varepsilon$ cover $\partial E$. Now the sum of the diameters of these covering sets is at most $p\left(E_{v}\right)+2 v \cdot\left[2 \cdot p\left(E_{v}\right) / \varepsilon\right]$, and we are done, since $p\left(E_{v}\right) \leq C_{T} \cdot a\left(E_{v}\right) \leq C_{T} \cdot a(E)$, and $v$ can be chosen arbitrarily small.

Now Theorem 4.2 is an immediate consequence of Lemmas 4.3 and 4.4.

\section{Quantitative Upper Bounds in the General Case}

In this section we give quantitative results on upper bounds depending on the original set.

In Theorem 3.1 we proved that $C_{U}<5.2 / 4=1.3$ if $U$ is a square, and, as we mentioned in the introduction, T. Keleti conjectures that $C_{U}=1$ for the square. We have seen in the introduction that $C_{U}$ can be arbitrarily large. We show, however, that for convex sets, $C_{U}$ can be estimated from above, depending on some parameters of $U$.

In [2] the following is proved. A bounded subset $F$ of the plane with diameter $D$ is said to be $r$-star-shaped if it contains a disc with radius $r D$ such that for each point $p$ of the disc and for every $q \in E$, the closed line segment $[p, q]$ is contained in $F$. If $E \subset \mathbb{R}^{n}$ is a (possibly infinite) union of $r$-star-shaped sets with diameter $D$, then $r(E) \leq C(n, r) / D$, where

$$
C(n, r)=\left(\frac{\sqrt{2}+1}{r}\right)^{n}\left(\left(1+\frac{\sqrt{2}+1}{r}\right)^{n}-1\right)
$$

[2, Corollary 12, p. 115].

Using Lemma 2.3, we can give a better estimate for $r(E)$ in the case $n=2$.

Proposition 5.1 Let $E$ be a bounded plane set which is a (possibly infinite) union of $r$-star-shaped plane sets of diameter D. Then $r(E) \leq 1 /\left(r^{2} D\right)$. 
Sketch of proof Let us call the convex hull of a given circle with radius $r D$ and a point a cone of ice cream of size $r D$. Note that each $r$-star-shaped set of diameter $D$ can be obtained as the union of ice cream cones of size $r D$. From this, similarly to the proof of Lemma 4.4, we may assume that $E$ is a finite union. By Lemma 2.3, it is enough to find a lower bound for $-\left\langle v^{E}(q), n^{E}(q)\right\rangle$ for all smooth points $q \in \partial E$. By the assumptions one can take a disc $F_{q}$ of radius $r D$ in $E$ such that the convex hull $E_{q}$ of $\{q\} \cup F$ is contained in $E$, and the diameter of $E_{q}$ is at most $D$. Note that $\partial E_{q}$ is not smooth at $q$. However, we define $n^{E_{q}}(q)$ by choosing one of the supporting lines of $E_{q}$ at $q$ that are tangent to the disc $F_{q}$, and then define $n^{E_{q}}(q)$ as the normal vector of this line.

It can be checked that $-\left\langle v^{E}(q), n^{E}(q)\right\rangle \geq-\left\langle v^{E_{q}}(q), n^{E_{q}}(q)\right\rangle$. Let $l_{q}$ denote the distance between $q$ and the center of $F_{q}$. An easy estimation shows that $-\left\langle v^{E_{q}}(q), n^{E_{q}}(q)\right\rangle$ is at least $r D \cdot \arcsin \left(r D / l_{q}\right)$. It is clear that $l_{q} \leq D$, so $-\left\langle v^{E_{q}}(q), n^{E_{q}}(q)\right\rangle \geq r^{2} D$. By using Lemma 2.3 we get that $r(E) \leq 1 /\left(r^{2} D\right)$.

Note that the estimate $r(E) \leq 1 /\left(r^{2} D\right)$ is better than $r(E) \leq C(n, r) / D$, where $C(n, r)$ is given by (3) since, for small $r, C(n, r)=O\left(1 / r^{4}\right)$.

It is a natural question whether or not $C_{U}=1$ holds for every convex $U$. As we have seen this in the Introduction, this is false even for triangles.

We do not know whether or not $C_{U}$ is bounded for convex sets. In the sequel we give upper bounds for $C_{U}$ for convex sets depending on some parameters of $U$. Our starting point is Lemma 2.6. Unfortunately, the right-hand side of (2) can be arbitrarily large. Indeed, if $U$ is a diamond of height 1 and having a small angle, then the scalar product of $v^{U}(q)$ and $n^{U}(q)$ can be arbitrarily small, while $r(U)$ is 4 . In order to get an estimate of $C_{U}$ for a convex set $U$, we shall use Proposition 5.1 together with a simple lower estimate for $r(U)$.

By plank we mean the region between two parallel lines. We denote by $w(U)$ the width of the set $U$ (recall that this is the width of the narrowest plank covering the set $U$ ). It is known that for convex sets, the quantities $r(U)$ and $1 / w(U)$ are of the same order of magnitude (see, e.g., [4]). For the sake of completeness, we prove a weaker statement here.

Proposition $5.22 / w(U) \leq r(U) \leq 8 / w(U)$ for any bounded convex set $U$ with nonempty interior.

Proof Clearly $p(U) \geq 2 \cdot \operatorname{diam} U$. On the other hand, take a plank with width $m$, and take a plank orthogonal to the previous one bounded by supporting lines. It is an easy observation that the distance of any two parallel supporting lines cannot exceed $\operatorname{diam} U$, which means that the area of the rectangle defined by these two planks is at most $\operatorname{diam} U \cdot w(U)$. Taking these two inequalities, we get that $p(U) / a(U) \geq$ $2 \cdot \operatorname{diam} U /(\operatorname{diam} U \cdot w(U))=2 / w(U)$, and this is the left-hand side of the inequality.

To get the right-hand side, first observe that $p(U) \leq 4 \cdot \operatorname{diam} U$. Indeed, we take the two planks defined in the previous paragraph; their intersection is a rectangle covering $U$ with sides of length at most $\operatorname{diam} U$. Since $U$ is convex, $p(U)$ cannot exceed the perimeter of any polygon covering $U$, and so we get the desired inequality $p(U) \leq 4 \cdot \operatorname{diam} U$. Now we have to estimate the area. Take two points $x$ and $y$ in 
$U$ with distance $\operatorname{diam} U$. The supporting lines of $U$ at points $x$ and $y$ can be chosen parallel. Now take two points $z$ and $w$ of $U$ in both sides of the line $x y$ such that the supporting lines of $U$ at $z$ and $w$ are parallel to the line $x y$. It is easy to see that the quadrilateral $x z y w$ is contained in $U$, and its area is at least $\operatorname{diam} U \cdot w(U) / 2$. Using these estimates, we finally get the desired inequality, since $p(U) / a(U) \leq 4$. $\operatorname{diam} U /(\operatorname{diam} U \cdot w(U) / 2)=8 / w(U)$.

Theorem 5.3 If $U$ is a bounded convex set with nonempty interior, then

$$
C_{U} \leq 18 \cdot \operatorname{diam} U / w(U) \text {. }
$$

Proof We shall reduce this theorem to Proposition 5.1 by finding a large ball in $U$. We use the notation of the proof of Proposition 5.2. We can suppose that the height belonging to the side $x y$ in the triangle $x y z$ is at least $w(U) / 2$ (otherwise we replace $z$ by $w)$. Now the radius of the inscribed circle in the triangle $x y z$ is $2 \cdot a(x y z) / p(x y z)$, and it is easy to see that $a(x y z) \geq \operatorname{diam} U \cdot w(U) / 4$ and $p(x y z) \leq 3 \cdot \operatorname{diam} U$, so the radius of the inscribed circle is at least $w(U) / 6$. This means that $U$ is $r$-starshaped with $r=w(U) /(6 \cdot \operatorname{diam} U)$. Theorem 5.1 and an easy calculation yield $C_{U} \leq$ $36 \cdot \operatorname{diam} U /\left(r(U) \cdot w(U)^{2}\right)$. From Proposition 5.2 we get $r(U) \cdot w(U) \geq 2$, and this finishes the proof.

We want to point out that for some convex sets, estimate (4) is far from the truth. In the next theorem we show that $C_{U} \leq 4$ for every rectangle $U$, while there are rectangles with diam $U / w(U)$ arbitrarily large.

Theorem 5.4 We have $C_{U} \leq 4$ for every rectangle $U$.

Proof According to Lemma 2.6, it is enough to estimate $-\left\langle v^{U}(q), n^{U}(q)\right\rangle$ for every smooth point $q \in \partial U$. Let $m \leq D$ denote the length of the sides of the rectangle $U$ (note that $m=w(U)$ ). If $q$ is on one of the shorter sides, then a simple calculation shows that the angle of the angular domain $\left\{\phi:-\left\langle v^{U}(q, \phi), n^{U}(q)\right\rangle=D\right\}$ is at least $\pi m /(4 D)$. Consequently, in these points, $\left\langle-v^{U}(q), n^{U}(q)\right\rangle$ is at least

$$
\frac{D \cdot(\pi m /(4 D))}{2 \pi}=\frac{m}{8} .
$$

If $q$ is on one of the longer side, then a simple calculation shows that the angle of the angular domain $\left\{\phi:-\left\langle v^{U}(q, \phi), n^{U}(q)\right\rangle=m\right\}$ is at least $\frac{\pi}{4}$, so in these points, $-v^{U}(q) \cdot n^{U}(q) \geq m / 8$ also holds. By Lemma 2.6 we get that $C_{U} \leq 8 /(m \cdot r(U))$. From Proposition 5.2 we know that $r(U) \cdot m \geq 2$, and this completes the proof.

\section{References}

1. Bognár, M.: On the exterior linear measure. Acta Math. Hung. 53(1-2), 159-168 (1989)

2. Keleti, T.: A covering property of some classes of sets in $\mathbf{R}^{n}$. Acta Univ. Carol., Math. Phys. 39(1-2), 111-118 (1998)

3. Schweitzer contest in higher mathematics 1998. Mat. Lapok (N.S.) 7(1-2), 47-67 (1997)

4. Scott, P.R., Wah, A.P.: Inequalities for convex sets. J. Inequal. Pure Appl. Math. 1(1), Article 6 (2000) $6 \mathrm{pp}$. (electronic) 